\title{
ON A PROBLEM OF G. G. LORENTZ REGARDING THE NORMS OF FOURIER PROJECTIONS
}

\author{
BORIS SHEKHTMAN
}

(Communicated by William J. Davis)

Abstract. For any $0<\alpha<\frac{1}{2}$ we construct a sequence of integers $\left(\mu_{1}, \ldots, \mu_{n}, \ldots\right)$ such that the norms of Fourier projections

$$
F_{N}=\sum_{1}^{N} e^{i \mu_{j} \theta} \otimes e^{i \mu_{j} t}: C_{[-\pi, \pi]} \rightarrow C_{[-\pi, \pi]}
$$

grow as $N^{\alpha}$. This answers a question of Prof. G. G. Lorentz.

\section{INTRODUCTION}

Let $M=\left(\mu_{1}, \ldots, \mu_{n}\right) \subset \mathbf{N}$ be an increasing sequence of integers. Let $C_{[-\pi, \pi]}$ be the space of continuous functions $f$ on $[-\pi, \pi]$ such that $f(-\pi)=$ $f(\pi)$. For every $f \in C_{[-\pi, \pi]}$ let $F_{M} f$ be the Fourier projection of $f$ :

$$
F_{M} f=\frac{1}{2 \pi} \sum_{j=1}^{n}\left(\int_{-\pi}^{\pi} f(\theta) e^{i \mu_{j} \theta} d \theta\right) e^{i \mu_{j} \theta} .
$$

The celebrated solution of the Littlewood conjecture (cf. [2]) implies (cf. [3])

$$
C_{1} \log (\# M) \leq\left\|F_{M}\right\| \leq C_{2} \sqrt{\# M} .
$$

We use $\# M$ to denote the cardinality of $M$. Here and throughout this paper we use the letter $C$ with subscripts to denote positive constants that do not depend on $M$.

It is also well known (cf. [1], [3]) that the lower and upper bounds in (1) are attained with $M$ being an arithmetic and lacunary sequence, respectively:

Proposition 1. There exist constants $C_{3}, C_{4}, C_{5}, C_{6}$ such that

(a) if $M$ is an arithmetic sequence then $C_{3} \log (\# M) \leq\left\|F_{M}\right\| \leq C_{4} \log (\# M)$

(b) if $M=\left(\lambda_{1}, \ldots, \lambda_{n}\right)$ is such that $\left(\lambda_{k}+1\right) / \lambda_{k}>2$ then $C_{5} \sqrt{\# M} \leq$ $\left\|F_{M}\right\| \leq C_{6} \sqrt{\# M}$.

Received by the editors March 1, 1989 and, in revised form, May 7, 1989.

1980 Mathematics Subject Classification (1985 Revision). Primary 41A45, 42A55; Secondary 41A35, 42A10, 42A 20 . 
In the 1986 Edmonton conference on constructive approximation, Professor G. G. Lorentz posed the following:

Problem. Let $0<\alpha<\frac{1}{2}$ be a real number. Exhibit a sequence of integers $M=\left(\mu_{1}, \ldots, \mu_{n}, \ldots\right)$ so that

$$
\left\|F_{M_{n}}\right\| \asymp n^{\alpha} \text { as } n \rightarrow \infty,
$$

where $M_{n}=\left(\mu_{1}, \ldots, \mu_{n}\right)$.

In this note we solve the problem.

The idea of the construction is to combine the lacunary sequences and arithmetic progressions in such a way that the cardinality of the set of arithmetic sequences is sufficiently large to be a major factor in $\# M_{n}$, while the cardinality of the lacunary sequence determines the norms of $\left\{F_{M_{n}}\right\}$, thus achieving an arbitrary balance between the two.

For a positive number $a$ we use $\lfloor a\rfloor$ to denote the integer part of $a$. We will need the following simple estimates:

Proposition 2. Let $a>1$. There exist constants $C_{7}(a), C_{8}(a), C_{9}(a), C_{10}(a)$ such that

$$
C_{7}(a) n^{a+1} \leq \sum_{k=1}^{n}\left\lfloor k^{a}\right\rfloor \leq C_{8}(a) n^{a+1}
$$

$$
C_{9}(a) n \log n \leq \sum_{k=1}^{n} \log \left\lfloor k^{a}\right\rfloor \leq C_{10}(a) n \log n .
$$

Proof. To prove (2) it suffices to observe

$$
\sum_{1}^{n}\left\lfloor k^{a}\right\rfloor \leq \sum_{1}^{n}\left\lfloor n^{a}\right\rfloor=n\left\lfloor n^{a}\right\rfloor \leq n \cdot n^{a} .
$$

On the other hand

$$
\sum_{1}^{n}\left\lfloor k^{a}\right\rfloor \geq \sum_{\lfloor n / 2\rfloor}^{n}\left\lfloor k^{a}\right\rfloor \geq \sum_{\lfloor n / 2\rfloor}^{n}\left\lfloor\left(\frac{n^{a}}{2}\right)\right\rfloor \geq\left(\frac{1}{2}\right)^{a} \cdot \frac{1}{2} n \cdot n^{a}-1 .
$$

The proof of $(3)$ is identical:

$$
\begin{aligned}
& \sum_{1}^{n} \log \left\lfloor k^{a}\right\rfloor \leq \sum_{1}^{n} \log =\left\lfloor n^{a}\right\rfloor \leq n \log \left(n^{a}\right)=\text { an } \log n . \\
& \sum_{1}^{n} \log \left\lfloor k^{a}\right\rfloor \geq \sum_{\lfloor n / 2\rfloor}^{n} \log \left(\frac{n}{2}\right)^{2} \geq C_{9}(a) n \log n . \square
\end{aligned}
$$

\section{Construction}

Given an $\alpha: 0<\alpha<\frac{1}{2}$ let

$$
a=3 / 2 \alpha-1: a>2 .
$$


We start with a lacunary sequence of integers

$$
\Lambda=\left(\lambda_{1}, \lambda_{2}, \ldots, \lambda_{n}, \ldots\right)
$$

that satisfies

$$
\lambda_{m+1}-\lambda_{m}>\left\lfloor n^{a}\right\rfloor \quad \text { for all } m \geq n .
$$

(For instance $\lambda_{m}=m^{m}$ will do).

We now construct the set $M$ in an inductive manner.

Choose $\mu_{1}=\lambda_{1}$. On the $n$th step we introduce the first $n^{2}$ available integers out of the set $\Lambda$ (say $\left.\lambda_{m+1}, \ldots, \lambda_{m+n^{2}}\right)$ and include in addition

$$
\lambda_{m+n^{2}}+1, \quad \lambda_{m+n^{2}}+2, \ldots, \lambda_{m+n^{2}}+\left\lfloor n^{a}\right\rfloor \text {. }
$$

By (5) we have

$$
\lambda_{m+n^{2}}+\left\lfloor n^{a}\right\rfloor<\lambda_{m+n^{2}+1}
$$

Now our set consists of a lacunary set

$$
\Lambda_{n}=\left(\lambda_{1}, \ldots, \lambda_{m+n^{2}}\right)
$$

of length

$$
\# \Lambda_{n}=\sum_{k=1}^{n} k^{2}
$$

and of a set of arithmetic sequences $\mathscr{A}_{1}, \ldots, \mathscr{A}_{n}$ of length

$$
\# \mathscr{A}_{j}=\left\lfloor j^{a}\right\rfloor \text {. }
$$

Theorem. For the set $M$ constructed above let $F_{N}:=F_{M_{N}}$. There exist constants $C, C_{0}>0$ such that

$$
C_{0} N^{\alpha} \leq\left\|F_{N}\right\| \leq C N^{\alpha} .
$$

Proof. Let $N$ be an integer and let us assume that the number $\mu_{N}$ had been added to the set $M$ during the $(N+1)$ st step of the construction of the set $M$. Then

$$
\# \Lambda_{n}+\sum_{k=1}^{n}\left(\# \mathscr{A}_{k}\right) \leq N \leq \# \Lambda_{n+1}+\sum_{k=1}^{n+1}\left(\# \mathscr{A}_{k}\right) \text {. }
$$

Using (6), (7), and (2) we have

On the other hand

$$
\begin{aligned}
N & \geq \sum_{k=1}^{n} k^{2}+\sum_{j=1}^{n}\left\lfloor j^{a}\right\rfloor \\
& \geq C_{7}(2) n^{3}+C_{7}(a) n^{a+1} \\
& \geq C_{11} n^{a+1} \quad(\text { since } a>2) .
\end{aligned}
$$

$$
\begin{aligned}
N & \leq C_{8}(2)(n+1)^{3}+\sum_{j=1}^{n+1}\left\lfloor j^{a}\right\rfloor \\
& \leq C_{8}(2)(n+1)^{3}+C_{8}(a)(n+1)^{a+1} \\
& \leq C_{12} n^{a+1}
\end{aligned}
$$


Hence we have

$$
C_{11} n^{a+1} \leq N \leq C_{12} n^{a+1} .
$$

Next we estimate the norms $\left\|F_{N}\right\|$.

Notice that $M_{N}=\Lambda_{n} \cup\left(\bigcup_{k=1}^{n} \mathscr{A}_{k}\right) \cup \Lambda^{p} \cup \mathscr{A}^{q}$ where $\Lambda^{p}$ is a lacunary sequence of length $p \leq(n+1)^{2}$ and $\mathscr{A}^{q}$ is an arithmetic sequence of length $q \leq$ $\left\lfloor(n+1)^{a}\right\rfloor$. Hence

$$
\left\|F_{N}\right\|=\left\|F_{\Lambda_{n} \cup \Lambda^{p}}+\sum_{k=1}^{n} F_{\mathscr{A}_{k}}+F_{\mathscr{A}} q\right\|,
$$

Since $\Lambda_{n} \cup \Lambda^{p}$ (is a lacunary set) we have (by Proposition 1(a))

$$
\begin{aligned}
\left\|F_{N}\right\| & \geq\left\|F_{\Lambda_{n} \cup \Lambda^{p}}\right\|-\left(\sum_{k=1}^{n}\left\|F_{\mathscr{A}_{k}}\right\|\right)-\left\|F_{\mathscr{A}} q\right\| \\
& \geq C_{5}\left[\#\left(\Lambda_{n} \cup \Lambda^{p}\right)\right]^{1 / 2}-C_{4} \sum_{k=1}^{n} \log \left(\# \mathscr{A}_{k}\right)-C_{4} \log \left(\# \mathscr{A}_{q}\right) \\
& \geq C_{5}\left(\left(\sum_{k=1}^{n} k^{2}\right)+p\right)^{1 / 2}-C_{4} \sum_{k=1}^{n} \log \left\lfloor k^{a}\right\rfloor-C_{4} \log \left\lfloor q^{a}\right\rfloor \\
& \geq\left[C_{5} C_{7}(2) n^{3}\right]^{1 / 2}-C_{4} C_{10}(a) n \log n-C_{4} \cdot a^{2} \log (n+1) \\
& \geq C_{14} n^{3 / 2} .
\end{aligned}
$$

Similarly

$$
\left\|F_{N}\right\| \leq\left\|F_{\Lambda_{n} \cup \Lambda^{p}}\right\|+\sum_{k=1}^{n}\left\|F_{\mathscr{S}_{k}}\right\|+\left\|F_{\mathscr{A}} q\right\| \leq C_{13} n^{3 / 2} .
$$

Hence

$$
C_{14} n^{3 / 2} \leq\left\|F_{N}\right\| \leq C_{13} n^{3 / 2}
$$

Combining (9) and (10) we have

as well as

$$
\left\|F_{N}\right\| \leq\left(C_{13} n^{3 / 2}\right) \leq C_{13}\left(\frac{N}{C_{11}}\right)^{3 / 2(a+1)} \leq C N^{3 / 2(a+1)}=C N^{\alpha}
$$

$$
\left\|F_{N}\right\| \geq C_{14} n^{3 / 2} \geq C_{14}\left(\frac{n}{C_{12}}\right)^{3 / 2(a+1)} \geq C_{0} N^{\alpha} .
$$

\section{REFERENCES}

1. C. C. Graham and O. C. McGehee, Essays in commutative harmonic analysis, Springer-Verlag, 1979.

2. O. C. McGehee, L. Pigno and B. Smith, Hardy's inequality and the $L^{1}$ norm of exponential sums, Ann. of Math. 113 (1981), 613-618.

3. B. Shekhtman, On the norms of some projections, Banach Spaces, Lecture Notes in Math. Vol. 1166 (1984), 177-185.

Department of Mathematics, University of South Flordia, Tampa, Florida 33620 